\title{
BRANCH STABILISATION FOR THE COMPONENTS OF HURWITZ MODULI SPACES OF GALOIS COVERS
}

\author{
MICHAEL LÖNNE
}

\begin{abstract}
We consider components of Hurwitz moduli space of $G$-Galois covers and set up a powerful algebraic framework to study the set of corresponding equivalence classes of monodromy maps. Within that we study geometric stabilisation by various $G$-covers branched over the disc.

Our results addresses the problem to decide equivalence and stable equivalence algebraically. We recover a homological invariant, which we show to distinguish the equivalence classes of given boundary monodromy and Nielsen type, if the latter is sufficiently large in the appropriate sense.
\end{abstract}

\section{INTRODUCTION}

In this article we want to discuss at length some group theoretical aspects crucial to the study of connected components of Hurwitz spaces of coverings, which can be traced back to [Cleb72, Hur91.

In the narrow sense, the objective is the study of morphisms $p: C \rightarrow C / G=: C^{\prime}$ induced by an effective action of a finite group $G$. The existence of such an effective action provides information about the complex structure of the curve and also about the group. In the broader sense, we include branched covers over Riemann surfaces, usually with at least one boundary component.

Let us recall first that the geometry of the covering $p$ encodes several numerical invariants that are constant under deformation: the genus $g^{\prime}$ of the base $C^{\prime}$ and its number of boundary components, the number $d$ of branch points $y_{1}, \ldots, y_{d} \in C^{\prime}$ and the orders $m_{1}, \ldots, m_{d}$ of the local monodromies - strictly speaking as an unordered multi-set. These invariants form the primary numerical type and have been the object of intensive studies since long.

A refined invariant is obtained from the monodromy $\mu: \pi_{1}\left(C^{\prime} \backslash\left\{y_{1}, \ldots, y_{d}\right\}\right) \rightarrow G$ of the regular unramified cover given by restriction of $p$ to the complement of the branch points, $p^{-1}\left(C^{\prime} \backslash\left\{y_{1}, \ldots, y_{d}\right\}\right)$. Instead of keeping track only of the orders $m_{i}$ of the elements in $G$ associated to the local monodromies at the punctures $y_{i}$, we consider the multi-set of their conjugacy classes. This is given by the Nielsen type, cf. [Niel37, the class function $\nu$ which on each conjugacy class $\mathcal{C}$ in $G$ takes the cardinality of local monodromies in $\mathcal{C}$ as its value. The function $\nu$ can be characterised also without taking recourse to the quotient $C^{\prime}$. For each conjugacy class $\mathcal{C}$ just count the number of $G$ orbits on $C$ such that an element is in $\mathcal{C}$ which rotates a disc around some point of the orbit by the smallest possible angle.

While many groups have been shown to allow classification by primary numerical type and Nielsen type, we are interested into arbitrary groups and get the motivation by the progress in the case of genus stabilisation.

Date: May 1, 2019.

The present work was done in the framework of the ERC Advanced grant n. 340258, 'TADMICAMT' 
Let us briefly recall the main results in the case of free actions. There the second homology group $H_{2}(G)$ was shown

- to classify equivalence classes of unbranched $G$-coverings for abelian and metabelian groups Edm82, Edm83.

- to classify stable equivalence classes for every group [Liv85].

- to classify equivalence classes for every group if the genus $g^{\prime}$ is sufficiently large DT06.

An analogous result to the last in the case of non-free action was proved with F. Catanese and F. Perroni [CLP16], where the second homology group had to be replaced by a quotient $H_{2, \Gamma}$.

The case of branch stabilisation is more involved. While genus stabilisation corresponds to connected sum with a trivial $G$-cover over the torus, summing a branched cover does involve making non-trivial choices for the monodromies at the branch points. In fact we will stabilise by boundary connected sum with a punctured disc, but consider very general choices for the branch monodromies.

We will succeed following the above program in the following sense

- we classify various sets of equivalence classes of $G$-covers by elements of a monoid or a set with a monoid action.

- we classify certain sets of stable equivalence classes of $G$-covers by elements of a set, which can be distinguished by the primary numerical type, the Nielsen type and using $H_{2, \Gamma}$.

- we classify equivalence classes of $G$-covers by their stable equivalence classes, if the Nielsen type is sufficiently large.

For example we prove the algebraic version, Thm 4.9 of the following geometric result.

Theorem 1.1. Suppose $\Gamma$ is a union of conjugacy classes generating $G$, then there exists an integer $N$ such that

the number of equivalence classes of $G$-covers of the disc with local monodromies in $\Gamma$ and fixed Nielsen type $\nu$ is independent of $\nu$, if $\nu$ takes a value at least $N$ on each conjugacy class.

Let us give a short overview on the content of this article: In the next section we recall Hopf formula and explain its validity in the setting of crossed modules. We then take some care to motivate the definition of a $G$-crossed module taking a union $\Gamma$ of conjugacy classes into account, which gives rise to a finite abelian group which is later shown to be equal to $H_{2, \Gamma}$.

In section 3 we equip the set of isomorphism classes of $G$-covers with an algebraic structure of monoid. This provides the necessary tool to investigate the geometric notion of stable equivalence by algebraic means. In the following section we address the stabilisation of $G$-covers branched over the disc, and determine conditions on the Nielsen types, such that stabilisation is surjective, resp. bijective.

Section 5 considers various geometric generalisations of $G$-covers and their equivalence classes. The corresponding algebraic setting is then presented and explored.

In the final section 6 we revise the definition of tautological central extension and recall the definition of quotient $H_{2, \Gamma}$ of $H_{2}(G)$ from [CLP15. We will then see how it fits very well with the set-up of the previous sections and deduce a classification result for $G$-covers with sufficiently large Nielsen type. 


\section{ALGEBRAIC SETTING}

The formula of Hopf describes the second homology group $H_{2}(G, \mathbb{Z})$ as the kernel of a natural map associated to a given group $G$. To avoid unnecessary generality we restrict to the case of a finite group of order $n:=\operatorname{ord}(G)$. In that case there is a natural finite presentation of $G$ for any map $S \rightarrow G$, such that the image generates $G$ as a group. It is expressed in a short exact sequence

$$
1 \rightarrow R \rightarrow F \rightarrow G \rightarrow 1,
$$

where $F=F_{S}$ is the free group freely generated by elements of $S$ and $R=R_{S} \subset F$ is the free subgroup of relations.

The Hopf formula [Hopf42] then states

$$
H_{2}(G, \mathbb{Z})=\frac{R \cap[F, F]}{[F, R]} .
$$

The same information is conveyed in the following exact sequences

$$
\begin{gathered}
1 \rightarrow H_{2}(G, \mathbb{Z}) \rightarrow \frac{F}{[F, R]} \rightarrow \frac{F}{[F, F]} \times G \rightarrow 1 \\
1 \rightarrow H_{2}(G, \mathbb{Z}) \rightarrow \frac{R}{[F, R]} \rightarrow \frac{F}{[F, F]} \rightarrow \frac{F}{R[F, F]} \rightarrow 1
\end{gathered}
$$

However, there is another setting which also provides an approach to the second homology of $G$ using a free object, the category of $G$-crossed modules. Let us quickly recall the basic definition.

Definition 2.1 A group homomorphism $\partial: C \rightarrow G$ with an action

$$
\begin{aligned}
C \times G & \rightarrow C \\
(c, g) & \mapsto c^{g}
\end{aligned}
$$

is called a $G$-crossed module, if

XM1: $\partial$ is $G$-equivariant for the conjugation action of $G$ on itself,

$$
\partial\left(c^{g}\right)=(\partial c)^{g}=g^{-1}(\partial c) g, \quad \forall c \in C, g \in G .
$$

XM2: the Peiffer identities hold

$$
c a=a c^{\partial a}, \quad \forall a, c \in C .
$$

Example 2.2 If $S \subset G$ generates $G$ then

$$
\partial_{S}: \frac{F_{S}}{\left[F_{S}, R_{S}\right]} \longrightarrow G
$$

is a $G$-crossed module which is a free $G$-crossed module on the inclusion $S \rightarrow G$, cf. [Rat80]. If $S$ is invariant under conjugation the $G$-action is simply induced by

$$
\begin{aligned}
& G \times F_{S} \longrightarrow F_{S} \\
& (g, \hat{a}) \mapsto \widehat{g^{-1} a g}
\end{aligned}
$$

which readily gives the $G$-equivariance of $\partial_{S}$ :

$$
\partial_{S}\left(\hat{a}^{g}\right)=\partial_{S}\left(\widehat{g^{-1} a g}\right)=g^{-1} a g=g^{-1}\left(\partial_{S} a\right) g .
$$

We note that the denominator of the Hopf formula has been incorporated into the crossed module. So it remains to perform the intersection of its numerator, between a 
derived subgroup and a kernel. This can be done with lots of $G$-crossed modules with still the second homology of $G$ dropping out.

Theorem 2.3 ([ElPo86]). If $\partial: C \rightarrow G$ is a projective $G$-crossed module ( in particular if it is a free $G$-crossed module) with $\partial$ surjective, then

$$
H_{2}(G, \mathbb{Z})=\operatorname{ker} \partial \cap[C, C] .
$$

We will now define a $G$-crossed module depending on a union $\Gamma \subset G$ of conjugacy classes. To this end, we first relax the notion of $G$-crossed module, dispensing with the group structure of the domain.

Definition 2.4 A map $\varepsilon: Q \rightarrow G$ from a $G$-set $Q$ to the group $G$ is called an augmentation and $Q$ an augmented quandle if

AQ1: $\varepsilon$ is $G$-equivariant for the conjugation of $G$ on itself, ( $Q$ is a $G$-crossed set)

$$
\varepsilon\left(p^{g}\right) \quad=g^{-1} \varepsilon(p) g, \quad \forall p \in Q, g \in G
$$

AQ2: idempotency holds in the sense

$$
p^{\varepsilon(p)}=p, \quad \forall p \in Q .
$$

$G$-crossed modules are augmented quandles, but also, more importantly, the set $\Gamma$.

Example 2.5 The union of conjugacy classes $\Gamma \subset G$ is a $G$ set for the action by conjugation. Therefore the injection $\varepsilon: \Gamma \rightarrow G$ is $G$-equivariant in the sense of AQ1. Property AQ2 also holds since any element in $G$ is unchanged under conjugation with itself.

We do not want to recall the story of the notion of quandle here, but refer for this and more on augmented quandles to the source [Joy82. Instead we take the quickest path back to $G$-crossed modules. It leads via the following definition.

Definition 2.6 Let $Q$ be an augmented quandle then the adjoint group $\operatorname{Adj} Q$ is the group presented by

$$
\operatorname{Adj} Q=\left\langle e_{q}, q \in Q \mid e_{p} e_{q}=e_{q} e_{p^{\varepsilon(q)}}\right\rangle .
$$

It has a unique group homomorphism to $G$ compatible with the augmentation $\varepsilon$

$$
\partial_{Q}: \operatorname{Adj} Q \rightarrow G, \quad e_{q} \mapsto \varepsilon(q)
$$

since $\operatorname{Adj} Q$ has the universal property for quandle maps to groups. And it serves our purpose, thanks to

Proposition 2.7. Suppose $\varepsilon: Q \rightarrow G$ is an augmented quandle, then

$$
\partial_{Q}: \operatorname{Adj} Q \rightarrow G
$$

is a crossed module over $G$ with respect to the tautological action of $G$ on $\operatorname{Adj} Q$ induced by the action of $G$ on $Q$

$$
\begin{aligned}
\operatorname{Adj} Q \times G & \rightarrow \operatorname{Adj} Q \\
\left(e_{q}, g\right) & \mapsto e_{q}{ }^{g}=e_{q^{g}}
\end{aligned}
$$


Proof. First we check that $\partial_{Q}$ is $G$-equivariant:

$$
\partial_{Q}\left(e_{q}^{g}\right)=\partial_{Q}\left(e_{q^{g}}\right)=\partial_{Q}\left(\varepsilon_{Q}\left(q^{g}\right)\right)=\varepsilon\left(q^{g}\right)=g^{-1} \varepsilon(q) g=\partial_{Q}\left(\varepsilon_{Q}(q)\right)^{g}=\partial_{Q}\left(e_{q}\right)^{g} .
$$

Second we have to check the Peiffer identities:

$$
e_{p}^{\partial_{Q}\left(e_{q}\right)}=e_{p}^{\partial_{Q}\left(\varepsilon_{Q}(q)\right)}=e_{p}^{\varepsilon(q)}=e_{p^{\varepsilon(q)}}=e_{p * q}=e_{q}^{-1} e_{p} e_{q}
$$

In analogy to the Hopf formula we get an abelian group

$$
H(\Gamma, G) \quad:=\operatorname{ker} \partial_{\Gamma} \cap[\operatorname{Adj} \Gamma, \operatorname{Adj} \Gamma] .
$$

As we will see, that intersection is a proper quotient of the second homology in general.

\section{THE HURWITZ MONOID}

To associate to a $G$-cover of the punctured disc an algebraic object, we recall the following geometric set-up:

Let $D$ be the closed unit disc, $\left(p_{n}\right) \subset D$ a sequence of distinct points in the interior, $p_{0} \in \partial D$ a base point and let $\left(\gamma_{n}\right)$ be a geometric basis for $\pi_{1}\left(D \backslash\left(p_{n}\right), p_{0}\right)$.

Any $G$-cover of $D$ - connected or not - which is unbranched outside $p_{1}, \ldots, p_{d}$ has a well-defined monodromy map relative $p_{0}$. To the homotopy class of a closed path it assigns the unique bijection acting from the right on the fibre at $p_{0}$ which gives the same map as path-lifting.

Only after choosing a point $\tilde{p}$ in the fibre, i.e. for a pointed $G$-cover, a monodromy map $\mu$ with values in $G$ is well-defined in general1: Via the (left) $G$-action the fibre at $p_{0}$ is identified with $\{g \tilde{p} \mid g \in G\}$ and $\mu$ takes the value $g$ if path-lifting maps $\tilde{p}$ to $g \tilde{p}$.

From now we tacitly assume all $G$-covers and their isomorphisms to be pointed and only put an occasional (pointed) to remind the reader of this fact.

In these circumstances, the chosen datum associates to a $G$-cover a monodromy tuple of elements in $G$.

$$
\mu\left(\gamma_{1}\right), \ldots, \mu\left(\gamma_{d}\right)
$$

As in previous papers, see [CLP15, CLP16], we want to call such a tuple a Hurwitz vector of genus 0 according to

Definition 3.1 For any $d>1$ the set $G^{d}$ is a $\mathrm{Br}_{d}$-set by the well-known Hurwitz action of the braid group. An element of this $\mathrm{Br}_{d}$-set is called a $(0, d)$-Hurwitz vector, while an element of $\coprod G^{d}$ is simply called a Hurwitz vector of genus 0 .

Two Hurwitz vectors $v, w$ are braid equivalent if they belong to the same braid group orbit,

$$
v \approx w
$$

Since on the geometric side we only consider isomorphism classes of $G$-covers, i.e. up to $G$-equivariant (pointed) maps covering a map preserving $\left(p_{n}\right)$ as a set, we get a one to one correspondence

$$
\left\{G \text {-covers of } D \text {, branched outside } p_{1}, \ldots, p_{d}\right\} / \text { iso } \stackrel{1: 1}{=} G^{d} / \mathrm{Br}_{d} \text {. }
$$

\footnotetext{
${ }^{1}$ points in the same orbit under the center of $G$ determine $G$-equivariantly isomorphic covers, in particular the choice is superfluous in case of abelian $G$.
} 
Now on the algebraic side we have a natural composition $G^{d} \times G^{e} \rightarrow G^{d+e}$ given by juxtaposition which obviously is associative. It is equivariant under the inclusions $\mathrm{Br}_{d} \rightarrow \mathrm{Br}_{d+e}$ and $\mathrm{Br}_{e} \rightarrow \mathrm{Br}_{d+e}$, where in the second case a braid on strands one to $e$ is moved to the corresponding braid on strands $d+1$ to $d+e$. The resulting monoid is called the Hurwitz class monoid of $G$ :

$$
\mathcal{H}_{G}:=\coprod_{d \geqslant 0} G^{d} / \mathrm{Br}_{d}
$$

where the unit is understood to be represented by the empty tuple.

This composition can also be constructed on the geometric side. Given a pair of $G$-covers unbranched outside $p_{1}, \ldots, p_{d}$, respectively $p_{1}, \ldots, p_{e}$, there is a unique class of $G$-covers unbranched outside $p_{1}, \ldots, p_{d+e}$, which is isomorphic to the first over a regular neighbourhood of $\gamma_{1} \cup \cdots \cup \gamma_{d}$ and to the second over a regular neighbourhood of $\gamma_{d+1} \cup \cdots \cup \gamma_{d+e}$, pointed by the same point $\tilde{p}$ over $p_{0}$.

On a moments thought, we may restrict on the geometric side to $G$-covers with local monodromies in a union of conjugacy classes $\Gamma \subset G$ and get on the algebraic side a submonoid

$$
\mathcal{H}_{G, \Gamma}:=\coprod_{d \geqslant 0} \Gamma^{d} / \mathrm{Br}_{d} .
$$

We next define equivalence of $G$-covers with respect to stabilisation by a $G$-cover $C_{u}$ corresponding to some $u \in G^{e}$.

Definition 3.2 Two $G$-covers are called $C_{u}$-stably equivalent if composition of either with the same number $\ell$ of copies of $C_{u}$ yield isomorphic $G$-covers.

The corresponding elements $v, w \in \coprod G^{d}$ are called $u$-stably equivalent if $v u^{\ell} \approx w u^{\ell}$, for some $\ell$.

So $u$-stable equivalence of $v, w$ is equality in the Hurwitz class monoid $\mathcal{H}_{G}$ of elements represented by $v u^{\ell}, w u^{\ell}$ for some $\ell$. In fact we can push this further to an equality in some monoid of fractions, but we need to recall the evaluation map on $\mathcal{H}_{G}$ first.

Lemma 3.3. The evaluation map defined on $\coprod G^{d}$ with values in $G$

$$
v=\left(v_{1}, \ldots, v_{d}\right) \quad \mapsto \quad e v(v):=v_{1} \cdots v_{d} \in G
$$

has the following properties:

i) ev is a monoid homomorphism.

ii) ev is constant on braid group orbits.

iii) ev induces an monoid homomorphism on the monoid $\mathcal{H}_{G}$, also called evaluation.

The proof is easy and left to the reader.

Now $e v(u) \in G$ has finite order - say $n$ - so we deduce that the elements $u^{\ell n}$ are central in $\mathcal{H}_{G}$ thanks to the following lemma proved in [CLP15].

Lemma 3.4. If $v, w$ are Hurwitz vectors of genus 0 and $e v(v)=1 \in G$, then $v w$ and wv are braid equivalent.

In particular these elements form a central submonoid of $\mathcal{H}_{G}$, which is the same as a central multiplicative set. The following technical result will provide the existence of corresponding monoids of fractions and expose their relation with the enveloping group of $\mathcal{H}_{G}$, defined by the universal property, that every monoid homomorphism to a group factors uniquely through the monoid homomorphism to the enveloping group.

Proposition 3.5. Suppose $\mathcal{S} \subset \mathcal{H}_{G}$ is a submonoid, equal to $\mathcal{H}_{G}$ or central and generated by an element $u^{\ell}$ with ev(u) of order dividing $\ell$, then 
i) there is an equivalence relation on $\mathcal{H}_{G} \times \mathcal{S}$ given by

$$
(v, s) \quad \sim \quad\left(v^{\prime}, s^{\prime}\right) \quad \Longleftrightarrow \quad \exists \tilde{v} \in \mathcal{H}_{G}, \tilde{s} \in \mathcal{S}: v \tilde{r}=v^{\prime} \tilde{s}, s \tilde{r}=s^{\prime} \tilde{s}
$$

Equivalence classes in case $\mathcal{S}$ generated by $u^{\ell}$ are written $[v / s]_{u}$.

ii) there is a well-defined monoid structure on equivalence classes induced by multiplication in $\mathcal{H}_{G}$, which for $\mathcal{S}$ central is

$$
\left[\frac{v}{u^{n \ell}}\right]_{u}\left[\frac{w}{u^{m \ell}}\right]_{u}=\left[\frac{v w}{u^{(n+m) \ell}}\right]_{u} .
$$

This monoid is denoted by $\mathcal{H}_{G} \mathcal{S}^{-1}$.

iii) the total monoid of fractions $\mathcal{H}_{G} \mathcal{H}_{G}^{-1}$ together with the map is uniquely isomorphic to the enveloping group of $\mathcal{H}_{G}$

iv) the monoid of fractions associated to $u$ is isomorphic to the enveloping group if

$$
g \in G \quad \Longrightarrow \quad \exists v \in \mathcal{H}_{G}, n>0: g v=u^{n \ell} \in \mathcal{H}_{G}
$$

Proof. The first two claims follow from the following two properties, well-know from localisation of rings, see [Sko06].

- $s \in \mathcal{S}, v \in \mathcal{H}_{G} \Longrightarrow s \mathcal{H}_{G} \cap v \mathcal{S} \neq \varnothing$

- $s \in \mathcal{S}, v, w \in \mathcal{H}_{G}, s v=s w \Longrightarrow \exists s^{\prime} \in \mathcal{S}: v s^{\prime}=w s^{\prime}$

Both are immediate for $\mathcal{S}$ central. The argument in the case $\mathcal{S}=\mathcal{H}_{G}$ is only slightly more difficult. In fact we note that every element has a power that is central, since its evaluation in the finite group $G$ has finite order and by Lemma 3.4 .

$$
\begin{aligned}
s \in \mathcal{S}, v \in \mathcal{H}_{G} & \Longrightarrow \quad s v^{\operatorname{ord} e v(v)}=v^{\operatorname{ord} e v(v)} s \in s \mathcal{H}_{G} \cap v \mathcal{S} \\
s \in \mathcal{S}, v, w \in \mathcal{H}_{G}, s v=s w & \Longrightarrow s^{\operatorname{ord} e v(s)} v=s^{\operatorname{ord} e v(s)} w \\
& \Longrightarrow \quad v s^{\operatorname{ord} e v(s)}=w s^{\operatorname{ord} e v(s)}
\end{aligned}
$$

To get the last two claims we first notice that for the monoids of fractions $\mathcal{H}_{G} \mathcal{S}^{-1}$ by construction any monoid homomorphism to a group factors. Thus we only need to show that every element has an inverse. This is trivially true for the the total monoid of fraction. In the case of claim $i v$ ) the elements of $\mathcal{H}_{G}$ given by a single letter $g \in G$ have an inverse $\left[v / u^{n \ell}\right]$ by hypothesis. Since these elements generate $\mathcal{H}_{G}$ every element in the monoid has an inverse.

We followed this route of progressive abstraction to have a criterion for stably equivalence in terms of an equality in a monoid of fraction which even is a group in the cases we are mostly interested in:

Corollary 3.6. Suppose $u \in \mathcal{H}_{G}$ has ev $(u) \in G$ of order $\ell$, then the following are equivalent

i) $v, w \in \mathcal{H}_{G}$ are $u-$ stably equivalent

ii) $[v / 1]_{u}=[w / 1]_{u} \in \mathcal{H}_{G} \mathcal{S}^{-1}$

Obviously, the proof of the proposition works also to proof analogous claims for submonoids of the monoids $\mathcal{H}_{G, \Gamma}$. However we skip the details and only spell out the corresponding corollary.

Corollary 3.7. Suppose $u \in \mathcal{H}_{G, \Gamma}$ has ev $(u) \in G$ of order $\ell$ and $\mathcal{S}=\left\{u^{n \ell}\right\}$, then the following are equivalent

i) $v, w \in \mathcal{H}_{G, \Gamma}$ are $u$-stably equivalent

ii) $[v / 1]_{u}=[w / 1]_{u} \in \mathcal{H}_{G, \Gamma} \mathcal{S}^{-1}$ 
In particular, if we want to decide $u$-stable equivalence in $\mathcal{H}_{G, \Gamma}$ under the hypothesis that the monoid of fractions is a group, then we can do so in the finitely presented group $\operatorname{Adj} \Gamma$.

Proposition 3.8. Suppose $u \in \mathcal{H}_{G, \Gamma}$ has ev $(u) \in \Gamma$ of order $\ell$ and $\mathcal{S}=\left\{u^{n \ell}\right\}$ is a denominator set such that $\mathcal{H}_{G, \Gamma} S^{-1}$ is a group, then

$$
\Longleftrightarrow \quad \begin{aligned}
& v, w \in \Gamma^{d} \text { are } u-\text { stably equivalent } \\
& e_{v_{1}} \cdots e_{v_{d}}=e_{w_{1}} \cdots e_{w_{d}} \in \operatorname{Adj} \Gamma
\end{aligned}
$$

Proof. By the corollary above it suffices to show that the second claim of the proposition is equivalent to the second claim of the corollary. This follows since both groups involved are the enveloping group of the monoid $\mathcal{H}_{G}$, and the element involved are mapped to each other by the unique map provided by the universal property. For the monoid of fractions this was shown above, for the adjoint group it is proved by Kamada, Matsumoto [KM05]. It uses the fact that the universal properties of the enveloping group and the adjoint group provide mutually inverse group homomorphisms between the two.

\section{THE GENUS ZERO CASE}

We investigate the relation between equivalence and stable equivalence of (pointed) $G$-covers branched over the disc, i.e. with $g=0$. They will be shown to be equal for the $G$-covers with sufficiently 'rich' branching and detectable in monoids of fractions from the last section. This involves studying conditions on the Hurwitz vector $u$ such that stability holds for geometric stabilisation by the corresponding $G$-cover $C_{u}$ according to

Definition 4.1 We say that stability holds for geometric stabilisation by $C_{u}$, if there exists a positive integer $m$, such that

$$
C_{u}-\text { stable equivalence }=G-\text { cover equivalence }
$$

on the $G$-covers equivalent to covers obtained by $m$ iterations of the $C_{u}$-stabilisation.

The condition of the definition easily translates into a condition on the algebraic side. Stability holds if

$$
u \text { - stable equivalence }=\text { braid equivalence }
$$

on the set of tuples braid equivalent to some $v u^{m}$. We are thus bound to study properties of Hurwitz vectors of the last kind in more detail.

Before entering into the discussion of stability, let us review some important tools, giving the definitions and providing their basis properties.

Definition 4.2 On the set $\coprod G^{d}$ of Hurwitz vectors for the finite group $G$

i) the relation

$$
v \leqslant w \quad: \Longleftrightarrow \quad \exists u: v u=w
$$

is called the prefix order on $\coprod G^{d}$.

ii) the relation

$$
v \lesssim w \quad: \Longleftrightarrow \quad \exists u: v u \approx w
$$

is called the weak prefix order on $\coprod G^{d}$, and so is the induced order on $\mathcal{H}_{G}$.

iii) an element $v \in G^{d}$ is said to generate the subgroup $H \subset G$ if

$$
\langle v\rangle \quad:=\left\langle v_{1}, \ldots, v_{d}\right\rangle=H .
$$


Now let $\left\{\mathcal{C}_{i}\right\}$ denote the set of equivalence classes for conjugacy on $G$, then

$$
\mathbb{Z}(G / \sim)=\oplus_{i} \mathbb{Z} \mathcal{C}_{i} \quad \text { with } \quad \nu \leqslant \nu^{\prime} \quad: \Longleftrightarrow \quad \nu_{i} \leqslant \nu_{i}^{\prime}, \forall i
$$

is a free abelian group with a partial order. Note that more generally the free abelian group on a union $\Gamma$ of conjugacy classes of $G$ is naturally isomorphic to the abelianisation of the adjoint group for the conjugation quandle $\Gamma$

$$
\oplus_{\mathcal{C}_{i} \subset \Gamma} \mathbb{Z} \mathcal{C}_{i}=H_{1}(\operatorname{Adj}(\Gamma), \mathbb{Z}) .
$$

Next we recall the basic properties of the Nielsen type and the subgroup generated by a Hurwitz vector, but leave the proofs to the reader, see also [CLP15, CLP16].

Lemma 4.3. The Nielsen map defined on $\coprod G^{d}$ with values in $\oplus_{\mathcal{C}_{i}} \mathbb{Z} \mathcal{C}_{i}=\mathbb{Z}(G / \sim)$,

$$
\begin{aligned}
v=\left(v_{1}, \ldots, v_{d}\right) & \mapsto \nu(v):=\sum \nu_{i} \mathcal{C}_{i} \\
\nu_{i} & =\#\left\{j \mid v_{j} \in \mathcal{C}_{i}\right\}
\end{aligned}
$$

has the following properties:

i) $\nu$ is a monoid homomorphism.

ii) $\nu$ is constant on braid group orbits.

iii) $\nu$ induces a monoid homomorphism on $\mathcal{H}_{G}$ also called Nielsen map.

iv) the Nielsen map is order preserving for both prefix orders

$$
v \leqslant w \quad \Longrightarrow \quad v \lesssim w \quad \Longrightarrow \quad \nu(v) \leqslant \nu(w),
$$

Lemma 4.4. The map \langle\rangle$: \coprod G^{d} \rightarrow\{H \mid H \subset G\}$ to the subgroups of $G$ partially ordered by inclusion has the following properties:

i) \langle\rangle is order preserving for both prefix orders on $\coprod G^{d}$.

ii) \langle\rangle is constant on braid group orbits.

iii) $\left\langle>\right.$ induces an order preserving map on $\mathcal{H}_{G}$ with respect to the weak prefix order.

A special role will be given to the Hurwitz vector defined in terms of $\Gamma \subset G$

$$
u_{\Gamma}=(\underbrace{g_{1}, \ldots, g_{1}}_{\text {ord } g_{1}}, \ldots, \underbrace{g_{r}, \ldots, g_{r}}_{\text {ord } g_{r}}),
$$

where $g_{1}, \ldots, g_{r}$ is an enumeration of the elements of $\Gamma$. Its braid equivalence class is independent of choices thanks to Lemma 3.4, since ev $\left(u_{\Gamma}\right)=1_{G}$. The corresponding denominator set generated by $u_{\Gamma}$ will be denoted by $\mathcal{S}_{\Gamma}$.

In the sequel, our argument is motivated by the approach of Conway and Parker, see [FV91]. The first step is the first part of [MM99, Lemma 6.9].

Lemma 4.5. Suppose $g_{1}, g_{2}$ are conjugate elements of order $n$ in a finite group $G$ and $v$ is a Hurwitz vector which generates $G$, then

$$
v g_{1}^{n} \approx v g_{2}^{n}
$$

The second part of that lemma inspired our next result:

Lemma 4.6. Let $w$ be a Hurwitz vectors generating $G$ and $u$ a Hurwitz vector with entries in $\Gamma$, then

$$
\nu(w) \geqslant \nu\left(u_{\Gamma} u\right) \quad \Longrightarrow \quad \exists v: w \approx v u, v \text { generates } G
$$


Proof. Thanks to induction, it suffices to prove the claim in case $u$ consists of a single entry $g$. By hypothesis the conjugacy class of $g$ occurs so often that by the pigeonhole principle there is a $g_{1}$ conjugate to $g$ which occurs at least $n=\operatorname{ord} g+1$ times. By Hurwitz moves we can obtain a Hurwitz vector $w^{\prime} g_{1}^{n+1}$ braid equivalent to $w$, hence $w^{\prime} g_{1}$ generates $G$. We apply Lemma 4.5 and obtain $w \approx w^{\prime} g_{1} g^{n}$. Thus our claim follows for $u=g$ and $v=w^{\prime} g_{1} g^{n-1}$.

Now we are ready to prove a bunch of bijectivity results for certain map induced by stabilisation maps.

Lemma 4.7. Let $u$ be a Hurwitz vector and suppose $\nu_{0} \geqslant \nu\left(u_{\Gamma(u)}\right)$, then for all $n \geqslant 1$

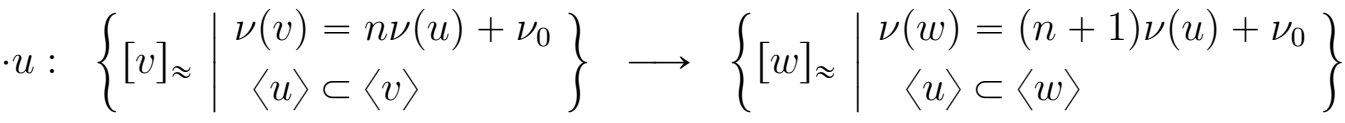

$$
\begin{aligned}
& {[v]_{\approx} \quad \mapsto \quad[v u]_{\approx}}
\end{aligned}
$$

is surjective and there exists $m=m\left(u, \nu_{0}\right)$, such that it is bijective for $n \geqslant m$.

Proof. To prove surjectivity we want to apply Lemma 4.6. Indeed, for $w$ with $[w]$ in the range, we let $G=\langle w\rangle$ and $\Gamma=\Gamma(u) \cap G$. Then $u$ has entries in $\Gamma$ and $\nu(w) \geqslant \nu(u)+\nu_{0} \geqslant \nu\left(u_{\Gamma} u\right)$, since $u_{\Gamma} \lesssim u_{\Gamma(n)}$. Hence we get the conclusion, that $[w]=\left[w^{\prime} u\right]$ with $\left\langle w^{\prime}\right\rangle=G \supset\langle u\rangle$.

Bijectivity follows from the fact, that an infinite chain of surjective maps between finite sets must eventually stabilise.

Proposition 4.8. Suppose $u_{\Gamma}$ generates $G$ invariably $\left(\right.$ i.e. $\left.\nu\left(u_{\Gamma}^{\prime}\right)=\nu\left(u_{\Gamma}\right) \Rightarrow\left\langle u_{\Gamma}^{\prime}\right\rangle=G\right)$, then there exists an integer $m=m(G)$ such that for

- any $\nu_{1} \geqslant \nu_{0} \geqslant m \nu\left(u_{\Gamma}\right)$ with $\nu_{0}, \nu_{1} \in \nu\left(\coprod \Gamma^{d}\right)$

- any Hurwitz vector $u$ with $\nu(u)+\nu_{0}=\nu_{1}$

juxtaposition of $u$ induces a bijective map

$$
\begin{aligned}
\cdot u:\left\{[v]_{\approx} \mid \nu(v)=\nu_{0}\right\} & \longrightarrow\left\{[v]_{\approx} \mid \nu(v)=\nu_{1}\right\} \\
{[w]_{\approx} } & \mapsto w u]_{\approx}
\end{aligned}
$$

Proof. Apply Lemma 4.7 and get $m=m\left(u_{\Gamma}, \nu\left(u_{\Gamma}\right)\right)$ such that $u_{\Gamma} \cdot$ is bijective for $n \geqslant m$. There are now Hurwitz vectors $u_{0}, u^{\prime}$ such that

$$
\nu\left(u_{0}\right)+m \nu\left(u_{\Gamma}\right)=\nu_{0}, \quad u_{0} u u^{\prime} \approx u_{\Gamma}^{k} \text { for some } k>0 .
$$

Then we get a factorisation of the bijective map $u_{\Gamma}^{k}$ :

$$
\left\{\nu(v)=m \nu_{\Gamma}\right\} \stackrel{\cdot u_{0}}{\longrightarrow}\left\{\nu(v)=\nu_{0}\right\} \stackrel{\cdot u}{\longrightarrow}\left\{\nu(v)=\nu_{1}\right\} \stackrel{\cdot u^{\prime}}{\longrightarrow}\left\{\nu(v)=k \nu_{\Gamma}\right\}
$$

Since $u_{\Gamma}$ generates $G$ invariantly, every element in the given sets generates $G$, hence Lemma 4.7 gives surjectivity of all three maps. Thus we conclude that each of the surjective maps, in particular $\cdot u$ is bijective.

Theorem 4.9. Suppose $u_{\Gamma}$ generates $G$, then there exists an integer $m=m(G)$ such that

i) for

- any $\nu_{1} \geqslant \nu_{0} \geqslant m \nu\left(u_{\Gamma}\right)$ with $\nu_{0}, \nu_{1} \in \nu\left(\coprod \Gamma^{d}\right)=\mathbb{N}(\Gamma / \sim)$

- any subgroup $H$ of $G$,

- any Hurwitz vector $u \in \coprod H^{d}$ with $\nu(u)+\nu_{0}=\nu_{1}$ 
juxtaposition of $u$ induces a bijective map

$$
\begin{aligned}
& \cdot u:\left\{[v]_{\approx} \mid \begin{array}{c}
\nu(v)=\nu_{0} \\
\langle v\rangle=H
\end{array}\right\} \rightarrow\left\{[v]_{\approx} \mid \begin{array}{c}
\nu(v)=\nu_{1} \\
\langle v\rangle=H
\end{array}\right\} \\
& {[w] \quad \mapsto \quad[w u]}
\end{aligned}
$$

ii) for any $\nu_{1}, \nu_{0} \geqslant m \nu\left(u_{\Gamma}\right)$ with $\nu_{0}, \nu_{1} \in \nu\left(\coprod \Gamma^{d}\right)$

$$
\#\left\{[v]_{\approx} \mid \nu(v)=\nu_{0}\right\}=\#\left\{[v]_{\approx} \mid \nu(v)=\nu_{1}\right\}
$$

Proof. Let $m$ be a common stability bound for the finitely many Hurwitz vectors $u_{\Gamma}^{\prime}$ with $\nu\left(u_{\Gamma}^{\prime}\right)=\nu\left(u_{\Gamma}\right)$. Then we can handle the claim of $i$ ).

In case $\nu_{0} \notin \nu\left(\coprod H^{d}\right)$, also $\nu_{1}$ is not contained, so both sides are empty sets and the claim is trivially true. Otherwise pick $u_{0} \in \coprod H^{d}$ with $\nu\left(u_{0}\right)=\nu_{0}$ and $u_{\Gamma}^{\prime} \in \coprod H^{d}$ with $\nu\left(u_{\Gamma}^{\prime}\right)=\nu\left(u_{\Gamma}\right)$ a multiple of $u_{\Gamma \cap H}$. Claim $i$ ) then follows as in the previous proofs.

The second claim is an immediate corollary, since the sets involved decompose over all possible subgroups $H$ of $G$ into the sets of the first claim and shown there to be bijective.

Definition 4.10 Call $m$ as in the theorem a $\Gamma$-stability bound.

Proposition 4.11. Suppose $v, w$ are Hurwitz vectors generating $G$ with entries in $\Gamma$, and $\nu(v) \geqslant \nu\left(u_{\Gamma}^{m}\right)$, where $m$ is a $\Gamma$-stability bound, then

$$
v \approx w \quad \Longleftrightarrow \quad[v / 1]=[w / 1] \in \mathcal{H}_{G, \Gamma} \mathcal{S}_{\Gamma}^{-1},
$$

Proof. We have only the prove the reverse implication. The first hypotheses on the right implies

$$
\begin{aligned}
v & \approx{ }_{u} w & & u_{\Gamma} \text {-stably } \\
\Longrightarrow v u_{\Gamma}^{\ell} & \approx w u_{\Gamma}^{\ell} & & \text { for some } \ell \\
\Longrightarrow & v \approx w & & \text { by theorem } 4.9
\end{aligned}
$$

In Corollary 3.6 we showed that elements with the same invariant are stably equivalent. In this proposition we show that elements with sufficiently high Nielsen class belong to the stable range, ie. where stable equivalence implies equivalence. The existence of a stability range expresses the fact that stability holds.

\section{GENERALISATIONS}

In this section we will aim for some generalisations going beyond the case of $G$-covers of the disc branched at a finite set of points up to $G$-equivariant covering isomorphisms.

Though we still want to understand stabilisation under 'geometric' composition with a $G$-cover $C_{u}$ over the disc corresponding to some $u \in G^{e}$, there are several different direction open to generalisation:

- include $G$-covers over surfaces of higher genus or higher number of boundary components, one boundary component is needed at least to perform geometric composition.

- modify the notion of isomorphism on the base: restrict the induced maps to preserve isotopy classes of appropriate geometric objects. 
- modify the notion of isomorphism on the fibres: restrict to maps preserving $G$-markings of a set of fibres.

To get the flavour of these generalisations, let us look at some examples and the corresponding algebraic structure.

Example 5.1 On the geometric side consider a Riemann surface $\Sigma=\Sigma_{g}^{1}$ of genus $g$ with one boundary component. Let $p_{0}$ be a point on the boundary, $\left(p_{n}\right) \subset \Sigma$ be a sequence of distinct points in the interior. Finally let $\alpha_{i}, \beta_{i}$ corresponding to handles, and a sequence $\left(\gamma_{n}\right)$ corresponding to the interior points be elements of a geometric basis for $\pi_{1}\left(\Sigma \backslash\left(p_{n}\right), p_{0}\right)$

As before any (pointed) $G$-cover of $\Sigma$, which is unbranched outside $p_{1}, \ldots, p_{d}$ has a monodromy map that gives rise to the monodromy tuple of elements in $G$

$$
\mu\left(\alpha_{1}\right), \mu\left(\beta_{1}\right), \ldots, \mu\left(\alpha_{g}\right), \mu\left(\beta_{g}\right), \mu\left(\gamma_{1}\right), \ldots, \mu\left(\gamma_{d}\right)
$$

In the present case, such tuples are naturally acted on by the mapping class group $\mathcal{M} a p_{g, d}^{1}:=\mathcal{M a p}\left(\Sigma_{g}^{1}\right)$, and we recall that elements of $G^{2 g+d}$ are called $(g, d)$-Hurwitz vectors, since the Cartesian product is considered as a $\mathcal{M} a p_{g, d^{-}}^{1}$ et. We get the one to one correspondence

$\left\{G\right.$-covers of $\Sigma$, branched outside $\left.p_{1}, \ldots, p_{d}\right\} /$ iso $\stackrel{1: 1}{=} G^{2 g+d} / \mathcal{M} a p_{g, d}^{1}$

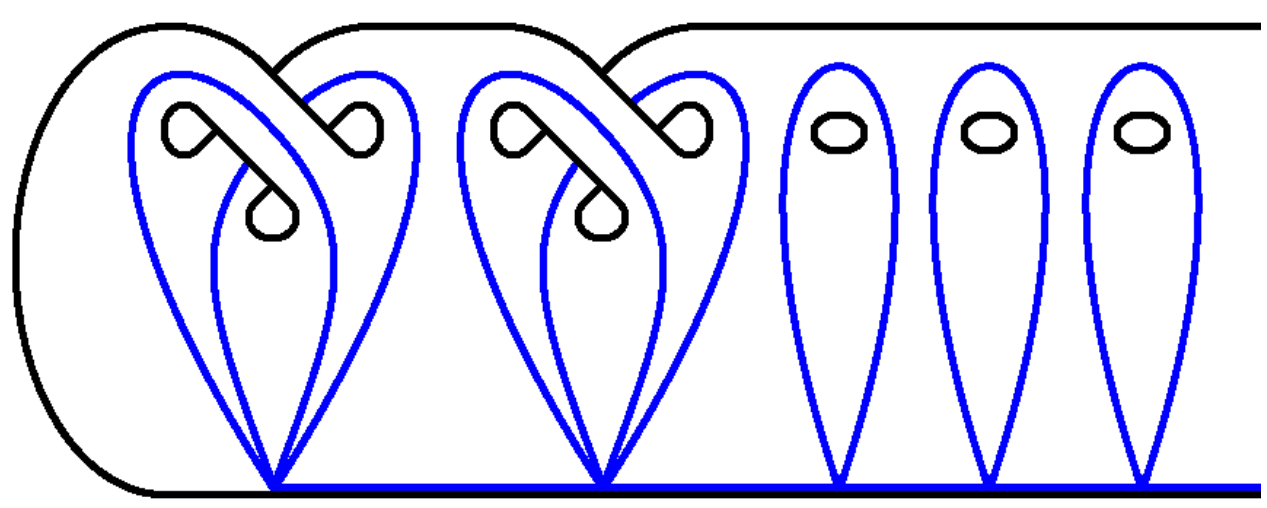

base with geometric basis

Example 5.2 Let us consider the $G$-covers of $\Sigma$ as in the previous example. However we define two $G$-covers to be isomorphic preserving chains if they are isomorphic via some $G$-equivariant covering map such that the induced map preserves the free isotopy classes of the $\alpha_{i}, \beta_{i}$.

Accordingly on the algebraic side we no longer have the action of the full mapping class group but rather that of the subgroup preserving the said isotopy classes. This subgroup can be identified with the mapping class group $\mathcal{M} a p_{0, d}^{g+1}$ of a subsurface $\Sigma^{\prime}$ of genus 0 with $d$ punctures and $g+1$ boundary components obtained from $\Sigma$ by cutting a regular neighbourhood of simple curves representing the given isotopy classes, thus

$$
\left\{G \text {-covers of } \Sigma \text {, branched outside } p_{1}, \ldots, p_{d}\right\} / \text { iso }_{\alpha, \beta} \quad \stackrel{1: 1}{=} G^{2 g+d} / \mathcal{M} a p_{0, d}^{g+1}
$$




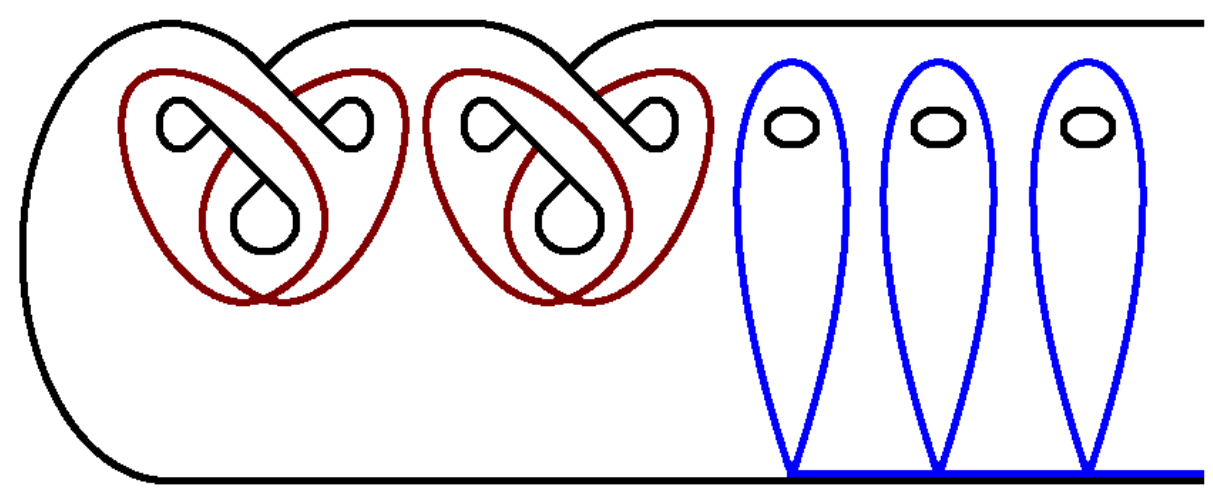

base with curves representing two chains

Example 5.3 For the third kind of generalisation we look again at $G$-covers of the disc $D$ equipped with distinct interior points $\left(p_{n}\right)$, a geometric basis $\left(\gamma_{n}\right)$ with respect to $p_{0}$ on the boundary. Let $q_{0}, \ldots, q_{k}$ be distinct points on the boundary with $q_{0}=p_{0}$. We introduce a $q$-marking to be given by $G$-equivariant maps $\ell_{i}$ from $G$ to the fibres over the $q_{i}$.

Then we get an extended monodromy map defined on the fundamental groupoid of the punctured base relative to the finite set of points $q_{i}$.

$$
\mu: \pi_{1}^{g r-o i d}\left(D \backslash\left\{p_{1}, \ldots, p_{d}\right\},\left\{q_{0}, \ldots, q_{k}\right\}\right) \longrightarrow G
$$

To the homotopy class of a path from $q_{i}$ to $q_{i^{\prime}}$ it associates the unique element $g$ in $G$ such that $g \ell_{i^{\prime}}(1)$ gives the same point in the fibre at $q_{i^{\prime}}$ as path lifting to $\ell_{i}(1)$.

The domain is a free groupoid on $d+k$ generators, and we end up with the one to one correspondence

$\left\{\right.$ marked $G$-covers of $D$, branched outside $\left.p_{1}, \ldots, p_{d}\right\} /$ iso $_{\text {marked }} \stackrel{1: 1}{=} \quad G^{k+d} / \mathrm{Br}_{d}$ The set $G^{k+d}$ in this case is a $\mathrm{Br}_{d}$-set as the Cartesian product of the trivial $\mathrm{Br}_{d}$-set $G^{k}$ and the $\mathrm{Br}_{d}$-set $G^{d}$ of Hurwitz vectors of genus 0 .

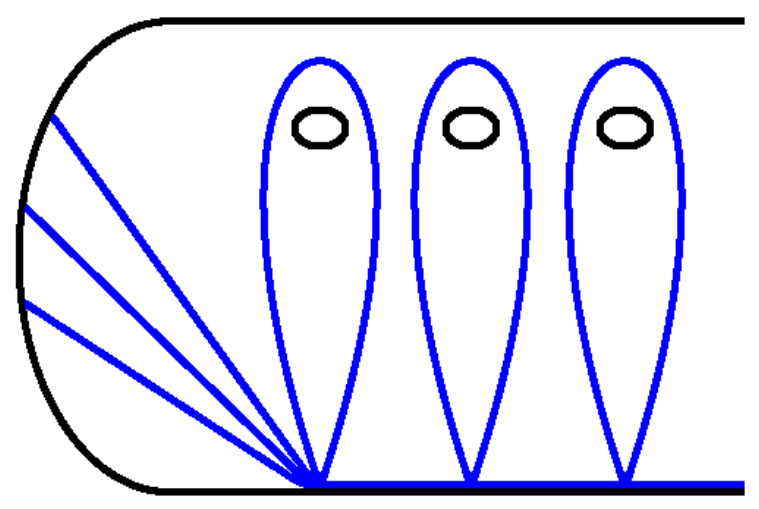

base with geometric base of groupoid

The common features of these generalisations - and others - on the algebraic side should be well noted:

- we classify orbits of $G$-tuples,

- the action is by some geometrically distinguished mapping class group,

- $\mathrm{Br}_{d}$ acts as the mapping class group of a suitable neighbourhood of $\gamma_{1}, \ldots, \gamma_{d}$. 
They will allow to get an algebraic model for composition with $G$-covers of genus 0 on the geometric side, which relies on the notion in the following definition:

Definition 5.4 Let $M$ be a monoid and $S$ be a set together with a map

$$
\rho: S \times M \rightarrow S, \quad(s, m) \mapsto s m
$$

such that

$$
s\left(m_{1} m_{2}\right)=\left(s m_{1}\right) m_{2}, \quad s 1_{M}=s
$$

Then we will call $\rho$ a $M$-action and $S$ a $M$-set (instead of the more common $M$-act).

Let us formulate the hypothesis in more abstract terms

Proposition 5.5. Suppose there is an array of nested groups

$$
\begin{aligned}
& \mathcal{M}_{0} \subset \mathcal{M}_{1} \subset \mathcal{M}_{2} \cdots \cdots \quad \cdots \quad \mathcal{M}_{d} \cdots
\end{aligned}
$$

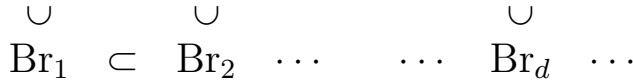

with $\mathcal{M}_{d}$ acting on $G^{r+d}$ such that as a $\mathrm{Br}_{d^{-}}$set $G^{r+d}$ is the Cartesian product of a trivial factor $G^{r}$ and the $\mathrm{Br}_{d^{-}}$set $G^{d}$ of Hurwitz vectors of genus 0 , then

i) $\coprod_{d} G^{r+d}$ is a $\coprod_{d} G^{d}$-set for concatenation,

ii) there is an induced action of the Hurwitz class monoid $\mathcal{H}_{G}$ on the $\mathcal{M}$-orbits

$$
\mathcal{Q}_{G, \mathcal{M}}:=\coprod_{d} G^{r+d} / \mathcal{M}_{d}
$$

Proof. Of course, the monoid $\coprod G^{d}$ is a $\coprod G^{d}$-set and the action is by concatenation. Thus the first claim follows from the observation that $\coprod_{d} G^{r+d}$ is an invariant subset.

For the second claim we have to show that the action on equivalence classes does not depend on the representatives. In fact

$$
x \mathcal{M}_{e}=y \mathcal{M}_{e}, v \operatorname{Br}_{d}=w \operatorname{Br}_{d} \quad \Longrightarrow \quad x v \mathcal{M}_{e+d}=y w \mathcal{M}_{e+d}
$$

since the action of $\mathrm{Br}_{d}$ on $G^{d}$ is the same as that on the second factor of $G^{r+e+d}=$ $G^{r+e} \times G^{d}$ via the action of $\mathrm{Br}_{e+d} \subset \mathcal{M}_{e+d}$ provided $\mathrm{Br}_{d}$ is considered as the subgroup of $\mathrm{Br}_{e+d}$ braiding only the last $d$ strands.

There is still one more essential feature:

- the number of local monodromies in each conjugacy class is invariant under the equivalence.

In fact a choice of a geometric free basis can be made in such a way, that the local monodromies correspond to the entries of a tuple in $G^{r+d}$ except for the first $s$ entries, with $s \leqslant r$. Hence in extension of the Nielsen type we get

Definition 5.6 The Nielsen map $\nu$ is defined on $G^{r+d}$ with values in $\oplus_{\mathcal{C}_{i}} \mathbb{Z} \mathcal{C}_{i}=\mathbb{Z}(G / \sim)$,

$$
\begin{aligned}
v=\left(v_{1}, \ldots, v_{r+d}\right) & \mapsto \nu(v):=\sum \nu_{i} \mathcal{C}_{i} \\
\nu_{i} & =\#\left\{j>s \mid v_{j} \in \mathcal{C}_{i}\right\}
\end{aligned}
$$

At this point we have collected enough information to turn back to the previous sections and see that their results generalise all along the way we have gone.

Remark 5.7 The action of $\mathcal{H}_{G}$ on $\mathcal{Q}_{G, \mathcal{M}}$ can be 'localised' at a denominator set $\mathcal{S}$ to yield a group action of the group $\mathcal{H}_{G} \mathcal{S}^{-1}$ on the set $\mathcal{Q}_{G, \mathcal{M}} \mathcal{S}^{-1}$. 
Moreover if $u \in \mathcal{H}_{G}$ has $e v(u) \in G$ of order $\ell$ and $\mathcal{S}=\left\{u^{n \ell}\right\}$, then the following are equivalent

i) $v, w \in \mathcal{Q}_{G, \mathcal{M}}$ are $u$-stably equivalent

ii) $[v / 1]_{u}=[w / 1]_{u} \in \mathcal{Q}_{G, \mathcal{M}} \mathcal{S}^{-1}$

We will say more about stabilisation. However, instead of pushing the generalisation to the limits, we fix $\Gamma=\Gamma_{G}=G \backslash\left\{1_{G}\right\}$ which contains all non-trivial elements of $G$ and generates $G$ invariably.

Lemma 5.8. Let $u$ be a Hurwitz vector and suppose $\nu_{0} \geqslant(r-s+1) \nu_{\Gamma}$, then

$$
\begin{aligned}
& \left\{v \mathcal{M} \mid \nu(v)=n \nu(u)+\nu_{0}\right\} \stackrel{\cdot u}{\longrightarrow}\left\{w \mathcal{M} \mid \nu(w)=(n+1) \nu(u)+\nu_{0}\right\} \\
& v \mathcal{M} \quad \mapsto \quad v u \mathcal{M}
\end{aligned}
$$

is surjective for $n>0$ and there exists $m=m\left(u, \nu_{0}\right)$, such that it is bijective for $n \geqslant m$.

Proof. The larger bound for $\nu_{0}$ is needed to make sure that for $w \in G^{r+d}$ its $\operatorname{tail}^{\tau} w \in G^{d}$ has $\nu\left({ }^{\tau} w\right) \geqslant \nu(u)+\nu_{\Gamma}$. Then we proceed as in the proof of Lemma 4.7 using Lemma 4.6 and that every Hurwitz vector with $\nu \geqslant \nu_{\Gamma}$ generates $G$ by our general assumption $\Gamma=\Gamma_{G}$

Theorem 5.9. Let $\Gamma=\Gamma_{G}$, then there exists an integer $m=m(G)$ such that for

- any $\nu_{1} \geqslant \nu_{0} \geqslant m \nu\left(u_{\Gamma}\right)$

- any Hurwitz vector $u$ with $\nu(u)+\nu_{0}=\nu_{1}$

juxtaposition of $u$ induces a bijective map

$$
\begin{aligned}
& \left\{[v]_{\mathcal{M}} \mid \nu(v)=\nu_{0}\right\} \stackrel{\cdot u}{\longrightarrow}\left\{[v]_{\mathcal{M}} \mid \nu(v)=\nu_{1}\right\} \\
& v \mathcal{M} \quad \mapsto \quad v u \mathcal{M}
\end{aligned}
$$

Proof. Apply Lemma 5.8 with $u=u_{\Gamma}$ and $\nu_{0}=(r+1) \nu_{\Gamma}$ to get $m$ and let $m=$ $m\left(u_{\Gamma}, \nu\left(u_{\Gamma}\right)\right)+r-s+1$ such that $u_{\Gamma}$ is bijective for $n \geqslant m$ on

$$
\left\{v \mathcal{M} \mid \nu(v)=n \nu_{\Gamma}\right\}
$$

There are now Hurwitz vectors $u_{0}, u^{\prime}$ such that

$$
\nu\left(u_{0}\right)+m \nu\left(u_{\Gamma}\right)=\nu_{0}, \quad u_{0} u u^{\prime} \approx u_{\Gamma}^{k} \text { for some } k>0 .
$$

Then we can conclude as in the proof of Prop. 4.8

This is the important task, since the analogous result for genus stabilisation will be applied in the argument for homological stability.

\section{The tautological LifT}

In the final section we revise the definition of tautological central extension and recall some information, see [CLP15], in particular the definition and properties of a quotient $H_{2, \Gamma}$ of $H_{2}(G)$ which proved to be crucial in our classification of curves with dihedral group of automorphisms.

We will then see how to recover that group in the set-up of the previous sections and deduce a classification result for (pointed) $G$-covers in the stable range.

Definition 6.1 Let $G$ be a finite group and let $F=F_{G}, R=R_{G}$ be as before. For any union of conjugacy classes $\Gamma \subset G$, define 
i) the tautological lift $G \rightarrow F_{G}$, which maps $g \mapsto \hat{g}$,

ii) the tautological map on $\coprod G^{d}: v=\left(v_{1}, \ldots, v_{d}\right) \mapsto \hat{v}_{1} \cdots \hat{v}_{d}$

iii) the normal subgroup $R_{\Gamma}$ normally generated by commutators in $[F, R]$ and tautological lifts of conjugacy relations for elements in $\Gamma$ :

$$
R_{\Gamma}=\left\langle\left\langle[F, R], \hat{a} \hat{b} \hat{c}^{-1} \hat{b}^{-1} \mid \forall a \in \Gamma, b \in G, c=b^{-1} a b\right\rangle\right\rangle
$$

( Note that the given elements generate this normal subgroup as subgroup. )

iv) the quotient group of $F$ by $R_{\Gamma}$

$$
G_{\Gamma}=F / R_{\Gamma} .
$$

v) the boundary homomorphism $\alpha: G_{\Gamma} \rightarrow G$, induced by $\hat{a} \mapsto a$ with kernel $K_{\Gamma}$.

Lemma 6.2. With the notation just introduced, $R_{\Gamma} \subset R$ and $K_{\Gamma}=R / R_{\Gamma}$. In particular $K_{\Gamma}$ is contained in the centre of $G_{\Gamma}$ and the short exact sequence

$$
1 \rightarrow \frac{R}{R_{\Gamma}} \rightarrow G_{\Gamma} \rightarrow G \rightarrow 1
$$

is a central extension.

Proof. $[F, R] \subset R$ because $R$ is normal in $F$. Moreover $\hat{a} \hat{b} \hat{c}^{-1} \hat{b}^{-1} \in R$ for any $a, b, c \in G$ with $a b=b c$, therefore $R_{\Gamma} \subset R$. By the definition of $\alpha$ we have that $K_{\Gamma}=\frac{R}{R_{\Gamma}}$. Finally, $K_{\Gamma}$ is in the centre of $G_{\Gamma}$ because $[F, R] \subset R_{\Gamma}$.

The tautological lift $G \rightarrow G_{\Gamma}, a \mapsto \hat{a}$ is not a group homomorphism in general, but every element in $G_{\Gamma}$ with image $g \in G$ can be written as $\hat{g} z=z \hat{g}$, with $z \in K_{\Gamma}$. Here, by abuse of notation, $\hat{g}$ denotes also the class of $\hat{g} \in F$ in $G_{\Gamma}=F / R_{\Gamma}$.

Remark 6.3 Let $\Gamma \subset G$ be the union of distinct conjugacy classes $C_{1}, \ldots, C_{t}$ and let $g_{1}, \ldots, g_{r}$ be the elements of $G \backslash \Gamma$, then the abelianisation $G_{\Gamma}^{a b}$ of $G_{\Gamma}$ is the free abelian group on $t+r$ generators

$$
G_{\Gamma}^{a b} \cong \mathbb{Z} C_{1} \oplus \cdots \oplus \mathbb{Z} C_{t} \oplus \mathbb{Z} g_{1} \oplus \cdots \oplus \mathbb{Z} g_{r}
$$

The Nielsen map $\nu$ on $\coprod \Gamma^{d}$ factors through the tautological map and the abelianisation as

$$
\coprod_{d} \Gamma^{d} \longrightarrow G_{\Gamma} \longrightarrow G_{\Gamma}^{a b} \longrightarrow \oplus_{i} \mathbb{Z} \mathcal{C}_{i}
$$

Definition 6.4 Let $\Gamma \subset G$ be a union of non-trivial conjugacy classes of $G$. We define

$$
H_{2, \Gamma}(G)=\operatorname{ker}\left(G_{\Gamma} \rightarrow G \times G_{\Gamma}^{a b}\right),
$$

where $G_{\Gamma} \rightarrow G \times G_{\Gamma}^{a b}$ is the morphism with first component the boundary map $\alpha$ and second component the abelianisation.

Let us recall from [CLP15 the precise relation between $H_{2}(G, \mathbb{Z})$ and $H_{2, \Gamma}(G)$.

Lemma 6.5. Let $G$ be a finite group and let $\Gamma \subset G$ be a union of nontrivial conjugacy classes. Write $G=\frac{F}{R}$ and $G_{\Gamma}=\frac{F}{R_{\Gamma}}$. Then, there is a short exact sequence

$$
1 \rightarrow \frac{R_{\Gamma} \cap[F, F]}{[F, R]} \rightarrow H_{2}(G, \mathbb{Z}) \rightarrow H_{2, \Gamma}(G) \rightarrow 1
$$

In particular $H_{2, \Gamma}(G)$ is abelian. 
Remark 6.6 The Schur multiplier is often interpreted as a cohomology group

$$
H^{2}(G, \mathbb{Q} / \mathbb{Z})
$$

which is algebraically dual to $H_{2}(G, \mathbb{Z})$. In case $\Gamma=G$ Moravec [Mo12] identified the group $H_{2, G}(G)$ with a subgroup of $H^{2}(G, \mathbb{Q} / \mathbb{Z})$ introduced by Bogomolov and justly called Bogomolov multiplier by Moravec.

The algebraic object from the previous to enter the stage is the adjoint group associated a quandle from Def.2. To emphasise its importance for the pair $G, \Gamma$ we give a more specific name:

Definition 6.7 Suppose $G$ is a finite group and $\Gamma \subset G$ a union of conjugacy classes, then the adjoint group for the quandle $\Gamma$

$$
\operatorname{Adj} \Gamma \quad:=\left\langle e_{g}, g \in \Gamma \mid e_{a} e_{b}=e_{b} e_{a^{b}}, a, b \in \Gamma\right\rangle
$$

is called the tautological crossed module of the pair $G, \Gamma$. The structural maps are

$$
\partial_{\Gamma}: e_{g} \mapsto g, \quad \operatorname{Adj} \Gamma \times G \rightarrow \operatorname{Adj} \Gamma:\left(e_{a}, g\right) \mapsto e_{a^{g}} .
$$

(It is a crossed module thanks to Prop 2.7.)

The two following results establish the close relation between the 'old' tautological central extension and the 'new' tautological crossed module.

Proposition 6.8. The map $e_{a} \mapsto \hat{a}$ for all $a \in \Gamma$ extends to an injective group homomorphism

$$
\operatorname{Adj} \Gamma \longrightarrow F / R_{\Gamma}
$$

with left inverse.

Proof. For the extension we only need to check that the relations of the domain map to $R_{\Gamma}$ :

$$
e_{a} e_{b} e_{a^{b}}^{-1} e_{b^{-1}} \quad \mapsto \quad \hat{a} \hat{b} \hat{c}^{-1} \hat{b}^{-1} \text {, with } c=a^{b}=b^{-1} a b .
$$

For the left inverse we first define a map $F \rightarrow \operatorname{Adj} \Gamma$ by

$$
\hat{h} \mapsto e_{g_{1}} \cdots e_{g_{r}}
$$

where for each $h \in G$ a unique factorisation $h=g_{1} \cdots g_{r}, r=r(h)$ has been chosen, with $r=1, g_{1}=h$ if $h \in \Gamma$. Since the composition of the two maps is the identity on $\Gamma$, it induces the identity map on $\operatorname{Adj} \Gamma$ if $R_{\Gamma}$ is in the kernel of this second map.

Let us note first, that by construction the map $F \rightarrow G$ factors through $\partial_{\Gamma}$. Accordingly $R$ maps to ker $\partial_{\Gamma}$. Now recall that for any crossed module $\partial: C \rightarrow G$ the kernel is central in $C$. In fact, conjugation by any element in the kernel is trivial by the Peiffer identity. We infer, that $R$ maps to the centre of $\operatorname{Adj} \Gamma$ and hence $[F, R]$ maps to the identity.

We complete the proof by showing that this is true also for the remaining elements generating $R_{\Gamma}$ :

$$
\begin{aligned}
\hat{g} \hat{h} & \mapsto \quad e_{g} e_{g_{1}} \cdots e_{g_{r}}=e_{g_{1}} e_{g^{g_{1}}} e_{g_{2}} \cdots e_{g_{r}}=e_{g_{1}} \cdots e_{g_{r}} e_{g^{h}} \\
\hat{h} \widehat{h^{-1} g h} & \mapsto \quad e_{g_{1}} \cdots e_{g_{r}} e_{g^{h}}
\end{aligned}
$$

Proposition 6.9. If $\Gamma$ generates $G$, then

$$
H_{2, \Gamma}=H(\Gamma, G):=\operatorname{ker} \partial_{\Gamma} \cap[\operatorname{Adj} \Gamma, \operatorname{Adj} \Gamma]
$$


Proof. Since the maps from the previous proposition induce the identity on the quotient $G$ and maps on the respective abelianisations, they also induce maps on the given groups. Therefore the group of the right is a subgroup of the other and it remains to show, that the kernel $N$ of $G_{\Gamma} \rightarrow \operatorname{Adj} \Gamma$ intersects $H_{2, \Gamma}$ trivially.

By the construction of the map, $N$ is normally generated by elements

$$
\hat{g}_{1} \ldots \hat{g}_{r} \hat{h}^{-1}, \quad \text { for all } h \notin \Gamma
$$

They all map to $1 \in G$, so they are central and generate a free abelian subgroup in $G_{\Gamma}$ of rank $|G \backslash \Gamma|$. The image in $\mathbb{Z}(G \backslash \Gamma)$ via $G_{\Gamma}^{a b}$ is of the same rank, since each generator is mapped to a standard generator. Thus $N$ maps injectively to $G_{\Gamma}^{a b}$ and thus intersects trivially with $H_{2, \Gamma}$.

To pursue the proof of the following classification result, we first need to introduce another Nielsen map.

Definition 6.10 The canonical extension of the Nielsen map $\mathcal{H}_{G} \rightarrow \oplus_{i} \mathbb{Z} \mathcal{C}_{i}$ to the localisation

$$
\begin{aligned}
\nu: \mathcal{H}_{G} \mathcal{S}_{\Gamma}^{-1} & \longrightarrow \oplus_{i} \mathbb{Z} \mathcal{C}_{i} \\
{\left[v / u_{\Gamma}^{\ell}\right] } & \mapsto \nu(v)-\ell \nu\left(u_{\Gamma}\right)
\end{aligned}
$$

is also called Nielsen map.

Theorem 6.11. Suppose $u_{\Gamma}$ generates $G$, then there exists an integer $m=m(G)$ such that for any $\nu_{0} \geqslant m \nu\left(u_{\Gamma}\right)$ with $\nu_{0} \in \nu\left(\coprod \Gamma^{d}\right)$

$$
\#\left\{[v]_{\approx} \mid \begin{array}{c}
\nu(v)=\nu_{0} \\
\langle v\rangle=G
\end{array}\right\}=\# H_{2, \Gamma} \times[G, G]
$$

Proof. First we note that there is an exact sequence induced from the central extension in Lemma 6.2

$$
H_{2, \Gamma} \quad \longrightarrow \quad \operatorname{ker} \nu \quad \longrightarrow \quad[G, G]
$$

Moreover, any $\left[v / u_{\Gamma}^{\ell}\right] \in \operatorname{ker} \nu$ gives two maps

$$
\cdot u_{\Gamma}, v: \quad\left\{[v]_{\approx} \mid \begin{array}{c}
\nu(v)=\nu_{0} \\
\langle v\rangle=G
\end{array}\right\} \rightarrow\left\{[v]_{\approx} \mid \begin{array}{c}
\nu(v)=\nu_{0}+\ell \nu_{\Gamma} \\
\langle v\rangle=G
\end{array}\right\}
$$

Both are bijective since we are above the stability bound, hence we can define a welldefined action

$$
[w]_{\approx}\left[v / u_{\Gamma}^{\ell}\right]=\left[w^{\prime}\right], \text { such that } w v \approx w^{\prime} u_{\Gamma}^{\ell} .
$$

Again using the fact, that we are in the stable range, the set is mapped injectively to the enveloping group $\operatorname{Adj} \Gamma$. This can be exploited to show that the action is free and transitive, because the action is now identified with multiplication inside $\operatorname{Adj} \Gamma$ by the subgroup $\operatorname{ker} \nu$.

While freeness is immediate, we are left to check, that any two elements $w, w^{\prime}$ are in one orbit: There is a unique element in the group $\operatorname{Adj} \Gamma$ which maps one to the other, which again we can write as $\left[v / u_{\Gamma}^{\ell}\right]$. Under the Nielsen map, it must map to 0 , since $\nu$ is a homomorphism on $\operatorname{Adj} \Gamma$. Thus this element is in $\operatorname{ker} \nu$ and we have also proved transitivity. The claim on the cardinality of the set is then obvious.

Rephrased in more geometrical terms the statement of the theorem tells us:

In the stable range connected (pointed) $G$-covers are classified up to equivalence by the Nielsen type, the evaluation, and an element in $H_{2, \Gamma}$. 
Do not miss the caveat: the homological information is not canonical, but depends on the choice of an element in each fibre of $\operatorname{Adj} \Gamma \rightarrow G \times \oplus_{i} \mathbb{Z} \mathcal{C}_{i}$.

\section{REFERENCES}

[CLP11] Catanese, F., Lönne, M., Perroni, F. Irreducibility of the space of dihedral covers of algebraic curves of fixed numerical type. Atti Accad. Naz. Lincei Cl. Sci. Fis. Mat. Natur. Rend. Lincei (9) Mat. Appl. 22 (2011), 1-19.

[CLP15] Catanese, F., Lönne, M., Perroni, F. The irreducible components of the moduli space of dihedral covers of algebraic curves, Groups Geom. Dyn. 9 (2015), 1185-1229.

[CLP16] Catanese, F., Lönne, M., Perroni, F. Genus stabilization for the components of moduli spaces of curves with symmetries, Algebr. Geom. 3 (2016), 23-49.

[Cleb72] Clebsch A., Zur Theorie der Riemann'schen Flächen. Math. Ann. 6, (1872), 216-230.

[DT06] Dunfield, N.M., Thurston, W.P. Finite covers of random 3-manifolds. Invent. Math. 166, (2006), 457-521.

[Edm82] Edmonds, A.L., Surface symmetry I, Michigan Math. J., 29 (1982), 171-183.

[Edm83] Edmonds, A.L., Surface symmetry II, Michigan Math. J., 30 (1983), 143-154.

[ElPo86] Ellis, G.J., Porter, T. Free and Projective Crossed Modules and the Second Homology Group of a Group. J. of Pure and Appl. Alg. 40 (1986), 27 - 31.

[FV91] Fried, M.D., Völklein, H. The inverse Galois problem and rational points on moduli spaces. Math. Ann. 290 (1991), 771-800.

[Hopf42] Hopf, H., Fundamentalgruppe und zweite Bettische Gruppe, Comment. Math. Helv. 14 (1942). 257-309.

[Hur91] Hurwitz, A.: Ueber Riemann'schen Flächen mit gegebenen Verzweigungspunkten. Math. Ann. 39, (1891), 1-61.

[Joy82] Joyce, D. A classifying invariant of knots, the knot quandle, J. Pure Appl. Algebra 23 (1982), 37-65.

[KM05] Kamada, S., Matsumoto, Y. Enveloping monoidal quandles, Top. and Appl. 146/147 (2005), 133-148.

[Liv85] Livingston, C. Stabilizing surface symmetries. Michigan Math. J. 32 (1985), 249-255.

[MM99] Malle, G.,Matzat B.H. Inverse Galois Theory, Monographs in Mathematics, Springer Verlag Berlin, 1999.

[Mo12] P. Moravec, Unramified Brauer groups of finite and infinite groups, Amer. J. Math. 134 (2012), 1679-1704.

[Niel37] Nielsen, J. Die Struktur periodischer Transformationen von Flächen. Danske Vid. Selsk. Math.-Fys. Medd. 15, (1937), 1-77.

[Rat80] Ratcliffe, J. Free and projective crossed modules. J. LMS (2) 22, (1980), no.1, 66-74.

[Sko06] Skoda, Z. Noncommutative localization in noncommutative geometry. Noncommutative Localization in Algebra and Topology, LMS Lecture Note Series, CUP, Cambridge, (2006), 220-310. 